\title{
Laparoscopic Approach to a Unilateral Adrenal Cyst in a Pediatric Patient
}

\author{
Christoph Castellani, MD, Georg Singer, MD, Manfred Ratschek, MD, Erich Sorantin, MD, \\ Florian Friedmacher, MD, Holger Till, MD \\ Department of Pediatric and Adolescent Surgery (Drs Castellani, Singer, Friedmacher, Till), Department of Pathology \\ (Dr Ratschek), Department of Radiology (Dr Sorantin), Medical University Graz, Graz, Austria.
}

\begin{abstract}
Introduction: Adrenal cysts are rare findings, with an age peak between the third and fifth decades of life. In adults, laparoscopic and retroperitoneoscopic approaches to these cysts have been described. In pediatric patients, however, reports of this pathology and its management are limited to a small number of case reports.

Case Report: A cystic mass was discovered in the left upper abdominal quadrant of a 12-year-old female patient during the diagnostic workup because of chronic back pain. Blood chemistry, parasitology, and tumor markers were negative, and the patient was scheduled for laparoscopy. Intraoperatively, the cyst was found to originate from the left adrenal gland. Firm attachments between the cyst and the adrenal gland required partial adrenalectomy. The operation and the postoperative course were uneventful, and the patient was discharged on the sixth postoperative day. The histologic workup revealed a true endothelial cyst of the adrenal gland.
\end{abstract}

Discussion: In the described case, laparoscopy was found to be a safe and feasible approach allowing definitive localization of the pathology and therapy within the same session.

Key Words: Laparoscopy, Adrenal cyst, Children, Abdominal cyst

Citation Castellani C, Singer G, Ratschek M, Sorantin E, Friedmacher F, Till H. Laparoscopic approach to a unilateral adrenal cyst in a pediatric patient. CRSLS e2014.002364. DOI: 10.4293/CRSLS.2014.002364

Copyright (C) 2014 SLS This is an open-access article distributed under the terms of the Creative Commons Attribution-Noncommercial-ShareAlike 3.0 Unported license, which permits unrestricted noncommercial use, distribution, and reproduction in any medium, provided the original author and source are credited.

Address correspondence to: Georg Singer, MD, Medical University Graz, Department of Pediatric and Adolescent Surgery, Graz, Austria, Tel: +43-316-385-13762, Fax +43-316-385-13775, E-mail: georg.singer@medunigraz.at.

\section{INTRODUCTION}

Adrenal cysts are rare findings in children as well as adults. ${ }^{1}$ Their incidence has been described as $0.06 \%$ in autopsy studies. ${ }^{2}$ They can be classified as parasitic ( $7 \%$ of cases), endothelial (approximately 45\%), epithelial (9\%), and pseudocyst (39\%). ${ }^{3}$ Although there are hypotheses concerning the etiology of epithelial cysts, the exact pathogenesis of endothelial cysts still remains unclear.

Epidemiologically, adrenal cysts are most commonly diagnosed between the third and fifth decades of life, ${ }^{1,4}$ with a female predominance (male/female ratio, 1:3). ${ }^{4}$ There is a tendency for adrenal cysts to occur on the right side of the body. ${ }^{1}$

Most adrenal cysts remain asymptomatic and are discovered incidentally. ${ }^{5}$ However, associations with abdominal pain 6 and pheochromocytoma-like symptoms due to compression of the adrenal gland ${ }^{7}$ have been reported.

In the literature, most reports deal with the management of adult adrenal cysts, and reports in children are scarce. ${ }^{8-11}$ Although common in adults, laparoscopic approaches to this disease in the pediatric population are limited to small case series. ${ }^{12,13}$ With this report, we add another rare case of laparoscopic management of a symptomatic pediatric adrenal cyst.

\section{CASE REPORT}

A 12-year-old girl presented to a tertiary center because of chronic back pain without a history of trauma. Routine magnetic resonance imaging was performed and revealed a cystic mass in the left upper abdominal quadrant (Figure 1). The patient was referred to our 


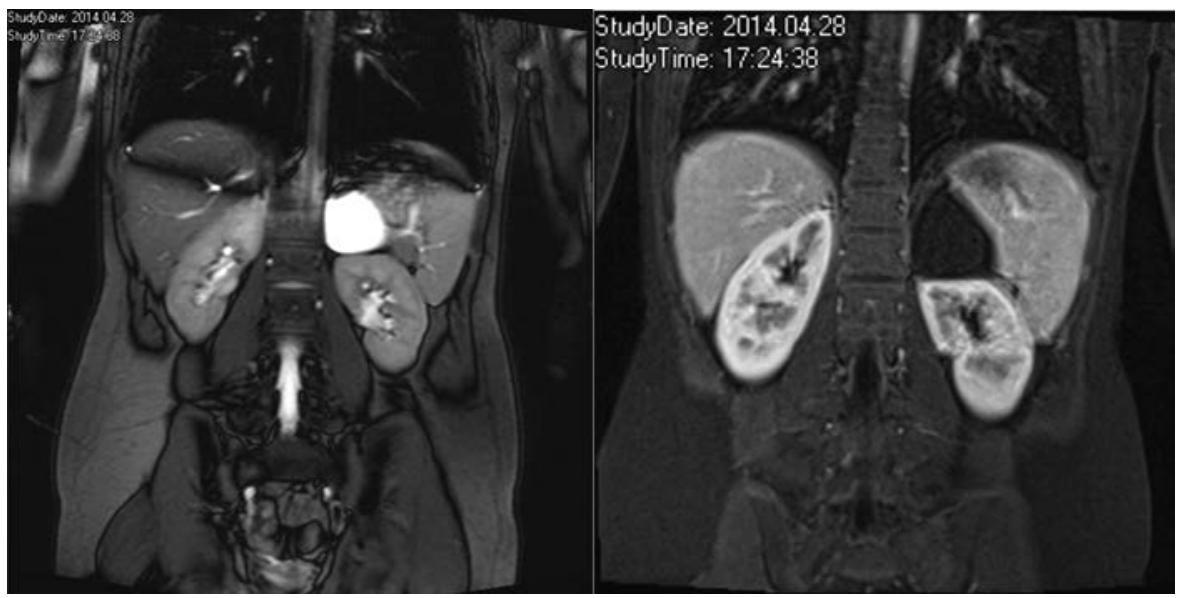

Figure 1. Magnetic resonance imaging with coronal planes revealing the localization of the cyst in the left upper quadrant in proximity to the left kidney and the spleen. (Left) T2 sequence. (Right) T1 sequence with contrast media without enhancement of the cyst.
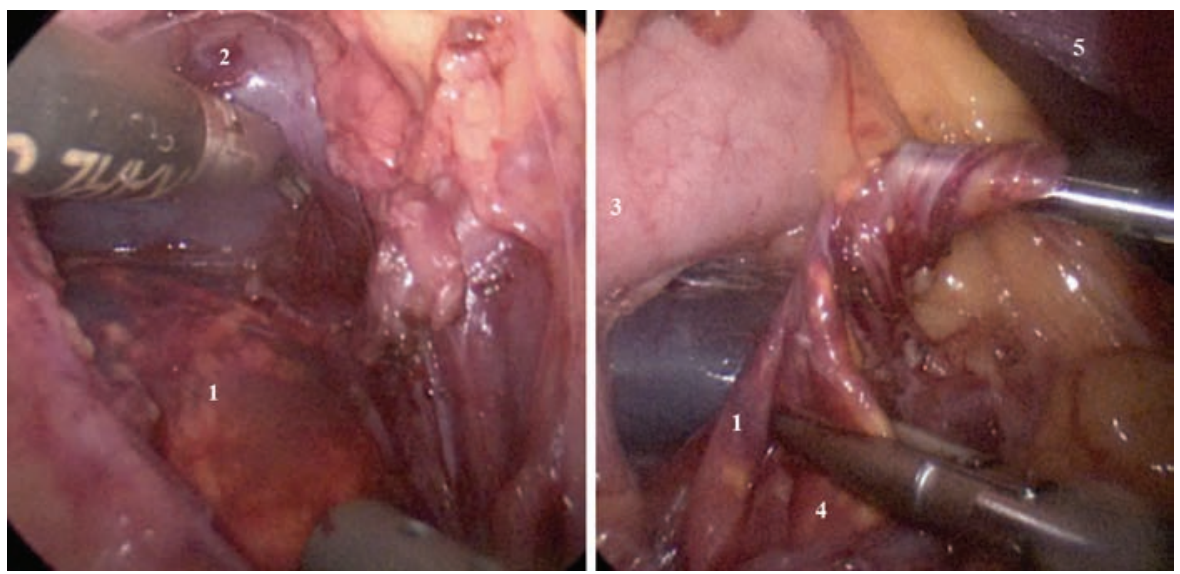

Figure 2. Intraoperative view of the cyst (5-mm laparoscope with $60^{\circ}$ angulation). (Left) Preparation and (right) resection of the cyst. $1=$ adrenal cyst, $2=$ transverse colon, $3=$ stomach, $4=$ adrenal gland, $5=$ spleen .

center for further diagnostics and treatment. Blood pressure and heart rate were within normal reference ranges. The patient underwent standard blood chemistry showing normal values for differential blood cell count, C-reactive protein, serial serum electrolytes, and renal and liver function. Urinalysis was negative. Further diagnostics included blood parasitology for Echinococcus and screening for tumor markers ( $\beta$-human choriogonadotropin, $\alpha$-fetoprotein, carcinoembryonic antigen, and human alkaline placenta phosphatase), all yielding normal values. Abdominal ultrasound gave no further details regarding etiology and localization of the cyst.

Subsequently, the patient was scheduled for laparoscopic exploration. The patient was put under general anesthesia and placed in the supine position with $30^{\circ}$ abduction of both hips. Laparoscopy was performed using a 4-trocar technique: an umbilical 5-mm trocar (with a 5-mm $60^{\circ}$ laparoscope), a $5-\mathrm{mm}$ trocar in the midline between the xiphoid and umbilicus, a 5-mm trocar in the middle of the upper left quadrant, and a left subxiphoid 3-mm trocar. Access to the lesser sac was gained identifying a retroperitoneal cystic mass near the caudal portion of the spleen (Figure 2). After opening the retroperitoneal space, the left adrenal gland became evident as the origin of the cyst. Firm attachments between cyst and gland required partial adrenalectomy using a LigaSure device (Covidien, Dublin, Ireland).

The further course was uneventful, and the patient could be discharged on the sixth postoperative day. Histologic analysis with hematoxylin and eosin staining showed an endothelial cyst of the adrenal gland (Figure 3). Immunohistochemistry staining was negative for cytokeratin 
(pan-cytokeratin-antibody staining for epithelial cells), D2-40 (mainly stains for lymphatic endothelium), calretinin (mainly stains for mesothelial cells), and CD31 (stains for panvascular endothelium) but positive for CD34 (stains for panvascular endothelium), confirming the diagnosis of an endothelial cyst (Figure 4).

\section{DISCUSSION}

Reports of true cysts of the adrenal gland are limited to small case series. ${ }^{8-11}$ Although several reports of laparoscopic and retroperitoneoscopic approaches to the adrenal gland in adults can be found, ${ }^{7,14}$ only a small number of reports deal with this technique in children. ${ }^{12,13}$ Here we describe the rare case of an endothelial cyst of the adrenal gland in a pediatric patient, to the best of our knowledge the first report of a laparoscopic approach in this age group.

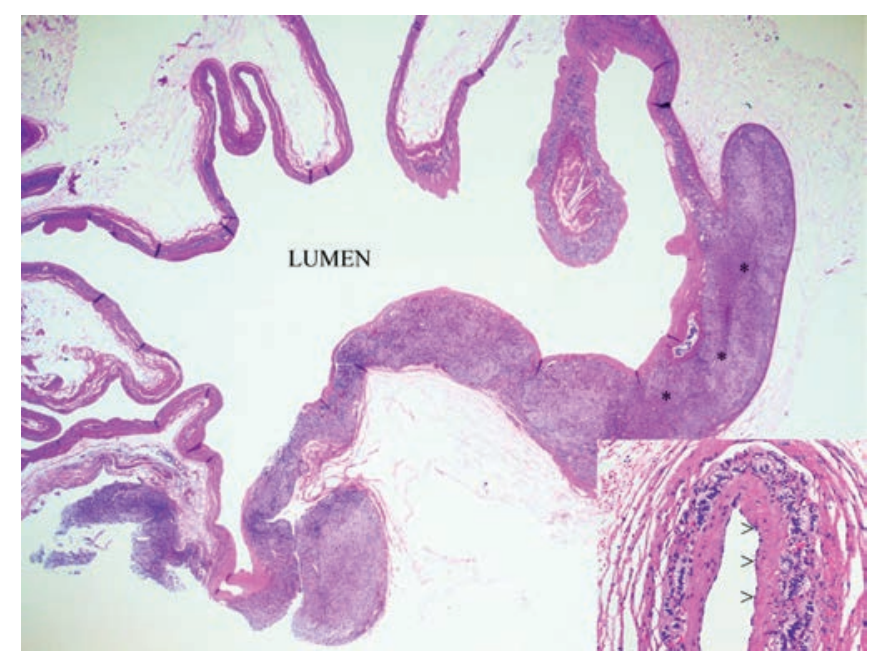

Figure 3. Hematoxylin and eosin stain of the cyst. The insert shows a close-up of the cyst with its endothelial lining (arrowheads) and tissue of the adrenal cortex (asterisks).
Most adrenal cysts remain asymptomatic and are discovered incidentally. ${ }^{15}$ However, there are frequent reports of patients presenting with flank pain, as in the case of our patient. ${ }^{6}$ Although it is reportedly superior to ultrasound in distinguishing between renal and adrenal origin, ${ }^{16}$ magnetic resonance imaging did not reveal the exact localization of the cyst in our patient. The abdominal ultrasound examination, however, did not provide any additional information. Despite the radiologic morphology, neither neoplasia nor Echinococcus cyst could be ruled out by imaging alone, prompting further laboratory analysis, as suggested in the literature. ${ }^{5}$ Pradeep et al ${ }^{1}$ have recommended further workup, including serial serum potassium, 24-hour urinary metanephrine, aldosterone-renin ratio, and a dexamethasone suppression test in patients with adrenal cysts. Govaerts et $\mathrm{al}^{5}$ suggested 24-hour urinary cortisol, serum potassium, a dexamethasone suppression test, serum adrenocorticotropic hormone levels, urinary catecholamine, and plasma serotonin for differential diagnosis among pheochromocytoma, hormonal active adenoma, and carcinoid. In our case, with a cyst of radiologically unclear origin, normal routine blood chemistry, negative tumor markers, normal blood pressure and heart rate, and no other clinical signs of a carcinoid (ie, flush, diarrhea), there was no clinical suspicion of these tumors, and therefore no hormone levels were determined.

A fine-needle aspiration biopsy would have been a diagnostic (and in some cases also therapeutic) option. However, it would have required additional general anesthesia in our pediatric patient. Additionally, hemorrhage after puncture has been reported as a frequent complication. ${ }^{1}$ Furthermore, it has been shown that clinical and radiologic findings alone, even if combined with fine-needle aspiration, cannot always provide a definitive diagnosis. ${ }^{4}$

With a diameter of $5 \mathrm{~cm}$ and an unclear origin as well as entity of the cyst, our patient met the criteria for surgical

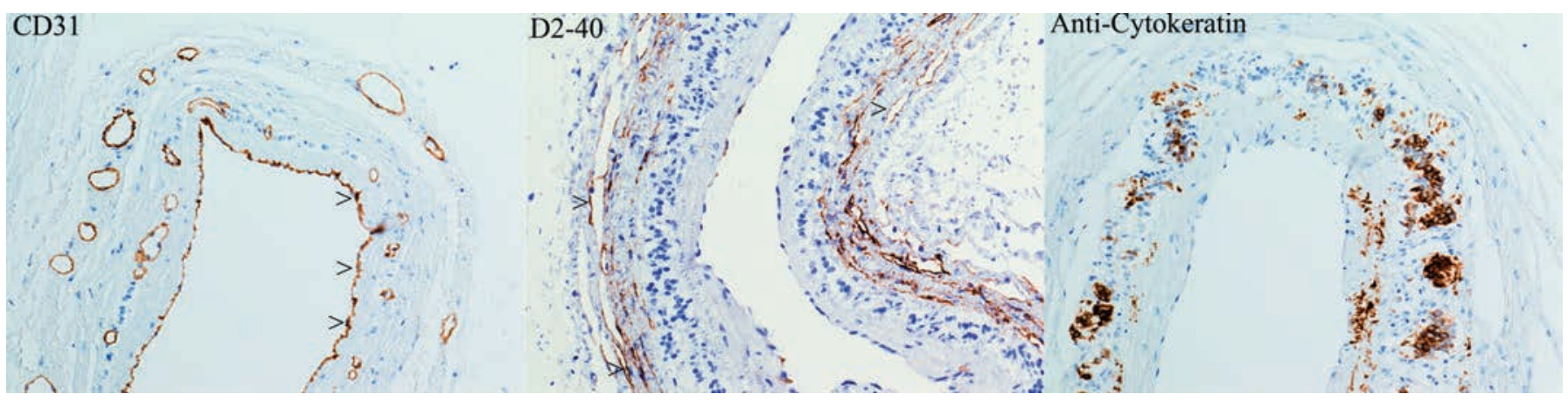

Figure 4. Immunohistochemical staining. The lining of the cyst stained positive for CD31 (left, arrowheads), negative for D2-40 (middle; note positive staining of lymphoid vessels [arrowheads]) and negative for anticytokeratin (right). 
removal recommended in the literature. ${ }^{1,17,18}$ Because of the unclear origin (abdominal or retroperitoneal), a laparoscopic approach was chosen. The localization of the cyst in the left upper quadrant prompted the placement of the ports as described. The operation was easily feasible, resulting in good clinical and cosmetic outcomes.

Histologic examination revealed an unlobulated cyst with the interior wall coated by flat endothelium-like cells. Possible differential diagnosis included mesothelium, flat epithelium, and vascular or lymphatic endothelial cells. Immunohistochemistry stained negative for cytokeratin, ${ }^{19}$ D2-40,20 and calretinin, ruling out epithelial cells, lymphatic endothelium, and mesothelium, respectively. CD31 stained positive and CD34 negative. Both are panvascular endothelial markers with different distributions, even within the same organ, ${ }^{20}$ but the positive reaction to CD31 proved the endothelial origin of the cyst resected in our case.

\section{CONCLUSIONS}

In the diagnostic workup of a pediatric adrenal cyst, neoplasia should be excluded as part of the differential diagnosis. Surgery is indicated in cases of large cysts with diameters $>5$ to $6 \mathrm{~cm}$ and in cases of unclear etiology. Laparoscopy proved to be a feasible and safe approach, allowing definitive localization of the pathology and therapy within the same session in the present patient.

\section{References:}

1. Pradeep PV, Mishra AK, Aggarwal V, Bhargav PR, Gupta SK, and Agarwal A. Adrenal cysts: an institutional experience. World J Surg. 2006;30:1817-1820.

2. Barron SH, Emanuel B. Adrenal cyst. A case report and a review ok the pediatric literature. J Pediatr. 1961;59:592-599.

3. Foster DG. Adrenal cysts. Review of literature and report of case. Arch Surg. 1966;92:131-143.

4. Limaiem F, Korbi S, Jedidi S, et al. Adrenal epithelial cyst: a case report. Pathologica. 2012;104:82-84.

5. Govaerts K, Van Eyken P, Verswijvel G, Van der Speeten K. A bronchogenic cyst, presenting as a retroperitoneal cystic mass. Rare Tumors. 2012;4:e13.

6. Wedmid A, Palese M. Diagnosis and treatment of the adrenal cyst. Curr Urol Rep. 2010;11:44-50.
7. Cao DH, Zheng S, Lv X, et al. Multilocular bronchogenic cyst of the bilateral adrenal: report of a rare case and review of literature. Int J Clin Exp Pathol. 2014;7:3418-3422.

8. Akata D, Haliloglu M, Ozmen MN, Akhan O. Bilateral cystic adrenal masses in the neonate associated with the incomplete form of Beckwith-Wiedemann syndrome. Pediatr Radiol. 1997;27:1-2.

9. Babin JP, Allain D, Demarquez JL, Bondonny JM, Leger H, Martin C. Adrenal cysts in the newborn. Apropos of 2 cases. Arch Fr Pediatr. 1977;34:130-142.

10. Morganti VJ, Anderson NG. Simple adrenal cysts in fetus, resolving spontaneously in neonate. J Ultrasound Med. 1991;10: $521-524$

11. Zivkovic SM, Jancic-Zguricas M, Jokanovic R, Nikezic M. Adrenal cysts in the newborn. J Urol. 1983;129:1031-1033.

12. Lopez PJ, Pierro A, Curry JI, Mushtaq I. Retroperitoneoscopic adrenalectomy: an early institutional experience. J Pediatr Urol. 2007;3:96-99.

13. Nerli RB, Reddy MN, Guntaka A, Patil S, and Hiremath M. Laparoscopic adrenalectomy for adrenal masses in children. JPediatr Urol. 2011;7:182-186.

14. Dong B, Zhou H, Zhang J, Wang Y, Fu Y. Diagnosis and treatment of retroperitoneal bronchogenic cysts: a case report. Oncol Lett. 2014;7:2157-2159.

15. Khan MR, Ajmal S, Saleem T. Giant adrenal endothelial cyst associated with acute and chronic morbidity in a young female: a case report. Cases J. 2009;2:8841.

16. Guo YK, Yang ZG, Li Y, et al. Uncommon adrenal masses: CT and MRI features with histopathologic correlation. Eur J Radiol. 2007;62:359-370.

17. Bellantone R, Ferrante A, Raffaelli M, Boscherini M, Lombardi CP, Crucitti F. Adrenal cystic lesions: report of 12 surgically treated cases and review of the literature. J Endocrinol Invest 1998;21:109-114

18. Darwish A, Nagaraj V, Mustafa MB, Al Ansari A. Adrenal cyst presenting as hepatic hydatid cyst. Case Rep Surg. 2013;2013: 150457.

19. Debus E, Moll R, Franke WW, Weber K, Osborn M. Immunohistochemical distinction of human carcinomas by cytokeratin typing with monoclonal antibodies. Am J Pathol. 1984;114:121-130.

20. Pusztaszeri MP, Seelentag W, Bosman FT. Immunohistochemical expression of endothelial markers CD31, CD34, von Willebrand factor, and Fli-1 in normal human tissues. J Histochem Cytochem. 2006;54:385-395. 